\title{
Presencia y Relaciones Anatomofuncionales del Ligamento Discomaleolar. Una Revisión de la Literatura
}

\author{
Presence and Anatomofunctional Relations of the Discomaleolar Ligament. A Literature Review
}

\author{
Carlos Salamanca $^{1,2}$; Fernando J. Dias ${ }^{1,3}$ \& Ramón Fuentes ${ }^{1,3}$
}

\begin{abstract}
SALAMANCA, C.; DIAS, F. J. \& FUENTES, R. Presencia y relaciones anatomofuncionales del ligamento discomaleolar. Una revisión de la literatura. Int. J. Morphol., 36(4):1356-1360, 2018.

RESUMEN: El Ligamento Discomaleolar es una estructura que no se describe en los textos clásicos de anatomía a pesar de ser descrito por primera vez por Rees, Pinto y luego por diversos autores como una estructura ligamentosa de tejido fibroelástico que conecta el cuello y el proceso anterior del martillo a través de la fisura petrotimpánica hasta la parte medioposterosuperior de la cápsula de la articulación temporomandibular (ATM), el disco articular y el ligamento esfenomandibular. Diversos estudios han reportado las implicaciones clínicas del ligamento discomaleolar y la relación entre los trastornos temporomandibulares con los síntomas asociados al oído medio. El objetivo de este estudio fue realizar una revisión sobre estudios que describen el ligamento discomaleolar y determinar si se considera una estructura independiente anatómica y funcionalmente y su relación con el oído medio. Se realizó una búsqueda de literatura científica identificó artículos donde se identifican las estructuras anatómicas asociadas a la articulación témporomandibular y el oído medio, fueron seleccionados 24 artículos según los criterios de selección. Los resultados obtenidos en los estudios seleccionados en esta revisión sugieren la existencia de una lámina o fascículo fibroso que conecta el disco de la ATM con el maléolo del oído medio, lo que validaría la presencia y relación entre el ligamento discomaleolar y los trastornos del oído medio.
\end{abstract}

PALABRAS CLAVE: Ligamento discomaleolar; anatomía de la ATM; Oído medio.

\section{INTRODUCCIÓN}

El ligamento discomaleolar, nombrado también como "ligamento de Pinto" o ligamento discomalear, es una estructura que no se describe en los textos clásicos de anatomía (Okeson, 2003; Latarjet \& Ruiz Liard, 2005), a pesar de ser descrito anatómica y funcionalmente por diversos autores (Pinto, 1962; Ioannides \& Hoogland, 1983; Rholin et al., 1985; Loughner et al., 1989; Ogutcen et al., 1993; Rodríguez Vázquez et al., 1993; Alves \& Figueiredo, 2010; Mérida-Velazco, 2007).

El ligamento discomaleolar, fue descrito por primera vez por Rees (1954) en una disección realizada en fetos y posteriormente detallada por Pinto, donde describe una estructura ligamentosa de tejido fibroelástico que conectaba el cuello y el proceso anterior del martillo a través de la fisura petrotimpánica hasta la parte medioposterosuperior de la cásula de la articulación temporomandibular, el disco articular y el ligamento esfenomandibular. Coleman (1970) estudió el ligamento discomaleolar en fetos y adultos, y describió una banda triangular de tejido conectivo, cuya base en continua con la porción posteromedial del disco y la cáp- sula articular que entra al oído medio a través de la fisura petrotimpánica.

En su desarrollo embrionario, Vásquez et al. (1997) describió un tracto fibroso que se originó en el mesénquima y se localizó craneal y lateral al cartílago de Meckel. Este tracto se estiró dorsalmente desde el área posterior del disco de la articulación temporomandibular hasta el oído medio a través de la parte más lateral de la fisura timpanoescamosa. Este tramo de tejido fibroso, que más tarde daría lugar al ligamento discomaleolar, fue unido al área de continuidad del cartílago de Meckel con el maléolo. La transformación del cartílago de Meckel en el ligamento esfenomandibular, el ligamento anterior del maléolo y el maléolo, con la excepción de su proceso anterior, determina su continuidad a través de la fisura tímpanoescamosa y explica la conexión entre el ligamento discomaleolar y el ligamento anterior del maléolo.

Toledo Filho et al. (1985) describen que las fibras del fascículo medio e inferior del ligamento anterior del martillo pasan a través de la fisura petrotimpánica para insertarse en la 
cápsula y en el disco de la articulación temporomandibular, mientras que Cesarini et al. (1991) observaron una conexión fibrosa entre la cápsula de la articulación temporomandibular y el cuello del martillo que consideraban corresponder a una porción del ligamento anterior de maléolo, sin embargo, Komori et al. (1986) describió el ligamento discomaleolar como una estructura independiente que se une individualmente cerca del cuello del maléolo.

Rodríguez-Vázquez et al. (1998), en un estudio con 20 cadáveres adultos, 10 masculinos y 10 femeninos, observaron que el ligamento discomaleolar era lateral al ligamento esfenomandibular. Se prolongó por encima y por dentro del disco que discurría lateralmente a través del ligamento esfenomandibular en dirección del cuello y base del proceso anterior del maléolo. El ligamento discomaleolar está unido ligeramente al ligamento esfenomandibular fuera del oído medio (porción yuxtaarticular) y también al interior (porción timpánica) y al margen de la fisura petrotimpánica. El ligamento discomaleolar sobrepasa la cavidad timpánica a través de la porción lateral de la fisura petrotimpánica.

Las disecciones de Pinto y Komori et al. y otras disecciones de adultos y fetos (Loughner et al., 1989; Rodríguez Vázquez et al., 1993; Ioannides \& Hoogland; Coleman) han establecido un enlace anatómico entre la articulación temporomandibular, el oído medio y la língula de ambos lados del canal anterior de la cuerda del tímpano (Canal de Huguier). El ligamento discomaleolar y el ligamento maleolar anterior pasan a través de este canal y conectan el proceso anterior del maléolo con el disco de la articulación temporomandibular (Hoshino, 1988; Toledo Filho et al.).

Esta relación entre el oído medio y la articulación temporomandibular indica que el ligamento discomaleolar tiene una gran importancia clínica, siendo descrita por algunos autores como una relación con los síntomas auditivos (Coleman; Ioannides \& Hoogland; Mérida-Velasco et al., 2012). Otros autores relatan que los trastornos termporomandibulares pueden provocar cambios en las estructuras del ligamento, incluyendo el ligamento discomaleolar, lo que podría provocar una tracción en el cuello del maléolo y luego causar síntomas como zumbido o hipoacusia (Pinto; Ionnides \& Hoogland).

A pesar de lo anterior, existe discusión entre los autores debido a que este ligamento ha sido nombrado y descrito con diferentes nombres y características, lo que ha llevado a la confusión (Alves \& Figueiredo; RodríguezVázquez et al., 1998). Entre los nombres que se le ha dado al ligamento está la banda discomaleolar (Rees), ligamento diminuto (Pinto), ligamento discomaleolar (Coleman), fascículo medio e inferior del ligamento anterior del maléolo
(Toledo Filho et al.), porción articular del ligamento anterior del martillo (Cesarani et al., 1991). El objetivo de este estudio fue realizar una revisión sobre estudios que describen el ligamento discomaleolar y determinar si se considera una estructura independiente anatómica y funcionalmente y su relación con el oído medio.

\section{MATERIAL Y MÉTODO}

Realizamos una búsqueda de literatura científica donde se identificaron las estructuras anatómicas asociadas a la articulación témporomandibular y el oído medio.

Criterios de inclusión: Artículos científicos publicados entre 1950 y 2017 en inglés, español y portugués, donde se identificaron las estructuras en seres humanos.

Criterios de exclusión: Artículos de diseño experimental o cuasiexperimental que fueron realizados en animales.

La selección de artículos fue realizada por dos investigadores de forma independiente; cuando existió controversia y diferencias en la aplicación de los criterios de selección, un tercer investigador realizó el análisis y final determinación para la incorporación o exclusión del estudio.

Estrategia de Búsqueda. Las bases de datos empleadas fueron MEDLINE, SCieLO, LILACS, ScienceDirect, Trip Database; Se realizó una revisión sistemática, sensible en la que se utilizaron términos MeSH y términos libres. Posteriormente se realizó una búsqueda específica en la que se añadieron los términos booleanos (AND/OR). Los límites fueron estudios realizados en seres humanos. El algoritmo de búsqueda en la base de datos MEDLINE fue: discomalleolar[All Fields] AND ("ligaments"[MeSH Terms] OR "ligaments"[All Fields] OR "ligament"[All Fields]) OR discomallear ligament. La búsqueda fue complementada con una revisión manual de las referencias de los artículos seleccionados.

\section{RESULTADOS}

Se encontró 68 artículos en las bases de datos MEDLINE, SciELO, LILACS, ScienceDirect y Trip Database. Se eliminaron 8 artículos duplicados, se realizó una revisión de los resúmenes dejando los artículos que aportaban información a la revisión según los parámetros descritos en los criterios de selección. Una vez finalizada la primera revisión, fueron seleccionados un total de 24 artículos, lo que se revisaron a texto completo (Fig. 1, Tabla I). 


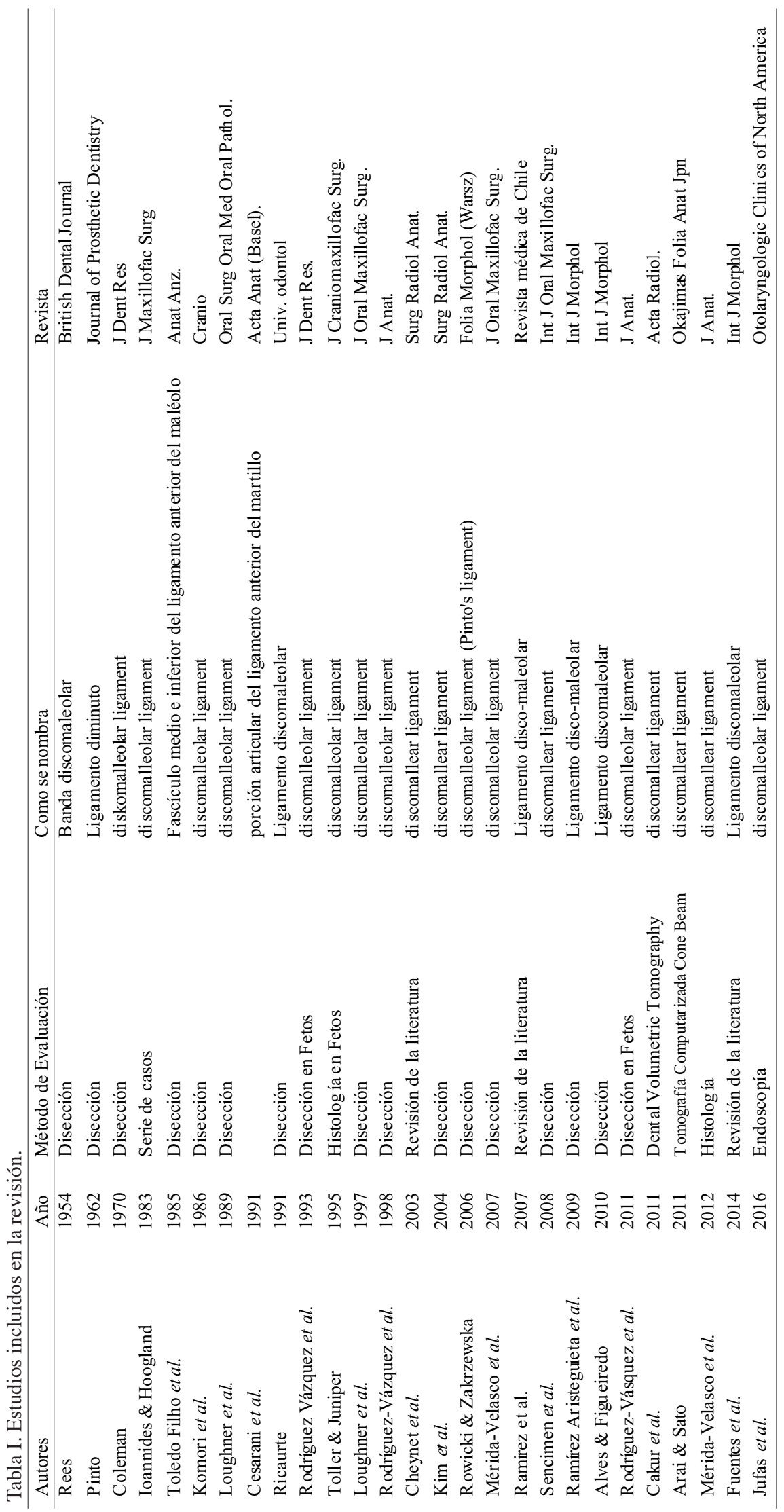

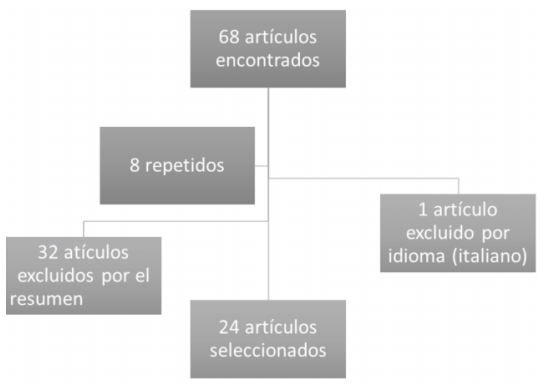

Fig. 1. Flujograma del proceso de búsqueda y selección de artículos para esta revisión.

\section{DISCUSIÓN}

Los resultados obtenidos en los estudios seleccionados en esta revisión sistemática indican la existencia de una lámina o fascículo fibroso que conecta el disco de la ATM con el maléolo del oído medio (Coleman; Rodríguez-Vásquez et al., 1993; Alves \& Figueiredo; Arai \& Sato, 2011; Mérida-Velasco et al., 2012).

A pesar de lo anterior, algunos autores afirman que este ligamento no es una estructura por sí sola, sino que es parte del ligamento anterior del maléolo (Burch, 1966; Toledo Filho et al.; Cesarani et al.) y otros que afirman que es una extensión del ligamento esfenomandibular en la cavidad timpánica (Burch) o un ligamento diminuto (Pinto). Tal confusión puede deberse a la dificultad que tiene su disección (RodríguezVásquez et al., 2011).

En su recorrido, el ligamento discomaleolar entra al oído medio a través de la porción más lateral de la fisura petrotimpánica. MéridaVelasco et al. (2012) describieron que existe una relación entre la cara lateral del ligamento discomaleolar y las ramas mediales del grupo posterior de la arteria timpánica anterior. El borde medial del ligamento discomaleolar presenta adherencias a 
la porción timpánica del ligamento esfenomandibular y ambos terminan en el cuello del maléolo por encima del proceso anterior, lo que concuerda con las observaciones realizadas en fetos humanos hechas por RodríguezVásquez et al. (1993).

En su desarrollo embrionario, estudios indican que el ligamento discolamaleolar está estrechamente relacionado con el cartílago de Meckel. Coleman escribió el ligamento en fetos, demostrando la estrecha relación entre el disco articular con el maléolo y el cartílago de Meckel, donde al ser examinado mediante histología se descubrió que algunas fibras del ligamento discomaleolar se unían a las paredes de la fisura petrotimpánica en el feto, lo cual fue confirmado en especímenes adultos. Rees describió que las fibras retrodiscales son el estrato superior de la zona bilaminar del disco articular y que representan la "banda discomaleolar de la vida fetal", que conecta el tendón del músculo pterigoideo al maléolo a través de la sutura tímpanoescamosa. Toller \& Juniper (1995) describieron que el ligamento discomaleolar derivó de la unión fibrosa del músculo pterigoídeo lateral al futuro maléolo, donde el musculo dejaba de estar con contacto con el ligamento a las 10 semanas de vida fetal.

Arai \& Sato realizaron una descripción histológica, donde encontraron fibras elásticas en la región anterior del ligamento discomaleolar que estaban conectadas a la región posterior del disco de la articulación temporamandibular, además se encontró en esta zona numerosas matrices extracelulares como la Bronectina y la Tenascina C, las cuales son importantes en la formación y disposición de las fibras colágenas.

Diversos estudios han demostrado las implicaciones clínicas del ligamento discomaleolar y la relación entre los trastornos temporomandibulares con los síntomas asociados al oído medio (Ramírez et al., 2007; RodríguezVásquez et al., 2011). Coleman, Komori et al. y Eckerdal (1991) indican que no hay evidencia de que la tracción del ligamento discomaleolar pueda desencadenar el movimiento de los huesos del oído medio. A su vez, RodríguezVásquez et al. (2011) describen que es posible un movimiento secuencial de los huesos del oído medio a través de la tracción del ligamento discomaleolar, proporcionando energía mecánica al maléolo, lo que está directamente relacionado con el grado de cierre de la fisura petrotimpánica durante el desarrollo, y por lo tanto, la adherencia entre el ligamento con el borde de la fisura petrotimpánica.

Entre los síntomas óticos reportados, autores afirman que el ligamento discomaleolar puede causar zum- bido de grandes proporciones y asociado a componentes musculares puede causar síntomas otológicos variados como hipoacusia, hiperacusia, vértigo, tinitus, sensación de oído tapado y otalgia (Parker \& Chole, 1995; Rodríguez-Vásquez et al., 1998; Ramírez et al.; Sencimen et al., 2008).

En general, el ligamento discomaleolar fue encontrado y descrito en la mayoría de los estudios, lo que indicaría que es un ligamento intrínseco de la articulación temporomandibular, debido a que tiene un origen embriológico demostrado, una disposición morfológica y función que lo distingue de la porción timpánica del ligamento esfenomandibular o del ligamento anterior del maléolo (Coleman; Rodríguez-Vásquez et al., 1993; Alves \& Figueiredo; Arai \& Sato; Mérida-Velasco et al., 2012).

Existe evidencia en la relación entre el ligamento discomaleolar y los trastornos del oído medio, pero existe discusión entre los autores en cómo este afecta directamente, por lo que es necesario más estudios que expliquen esta relación.

SALAMANCA, C.; DIAS, F. J.\& FUENTES, R. Presence and anatomofunctional relations of the discomaleolar ligament. A literature review. Int. J. Morphol., 36(4):1356-1360, 2018.

SUMMARY: The Discomaleolar Ligament is a structure that is not described in the classic texts of anatomy despite being described for the first time by Rees, Pinto and later by various authors as a ligamentous structure of fibroelastic tissue that connects the neck and the anterior process of the malleus through the petrotympanic cleft to the mid-superior part of the capsule of the temporomandibular joint (TMJ), the articular disc and the sphenomandibular ligament. Several studies have reported the clinical implications of the discomaleolar ligament and the relationship between temporomandibular disorders and symptoms associated with the middle ear. The aim of this study was to conduct a review of studies that describe the discomaleolar ligament and determine if an anatomically and functionally independent structure and its relation to the middle ear is considered. A search was performed of scientific literature identified articles where the anatomical structures associated with the temporomandibular joint and the middle ear were identified, 24 articles were selected according to the selection criteria. The results obtained in the studies selected in this review suggest the existence of a lamina or fibrous fascicle that connects the TMJ disc with the malleolus of the middle ear, which would validate the presence and relationship between the discomaleolar ligament and middle ear disorders.

KEY WORDS: Discomaleolar ligament; TMJ anatomy; Middle ear. 


\section{REFERENCIAS BIBLIOGRÁFICAS}

Alves, N. \& Figueiredo, D. N. Frequency of occurence of the discomalleolar ligament in the adult man. Int. J. Morphol., 28(2):515-8, 2010.

Arai, H. \& Sato, I. Anatomical study of the human discomallear ligament using cone beam computed tomography imaging and morphological observations. Okajimas Folia Anat. Jpn., 88(3):89-101, 2011.

Cakur, B.; Sümbüllü, M. A.; Durna, D. \& Akgül, H. M. Prevalence of the types of the petrotympanic fissure in the temporomandibular joint dysfunction. Acta Radiol., 52(5):562-5, 2011.

Cesarani, A.; Tombolini, A.; Fagnani, E. \& Domenech Mateu, J. M. The anterior ligament of the human malleus. Acta Anat. (Basel), 142(4):3136,1991.

Cheynet, F.; Guyot, L.; Richard, O.; Layoun, W. \& Gola, R. Discomallear and malleomandibular ligaments: anatomical study and clinical applications. Surg. Radiol. Anat., 25(2):152-7, 2003.

Coleman, R. D. Temporomandibular joint: relation of the retrodiskal zone to Meckel's cartilage and lateral pterygoid muscle. J. Dent. Res., 49(3):626-30, 1970 .

Fuentes, R.; Bucchi, C. \& Cantin, M. A Study of terms used in scientific literature to refer to the extracapsular ligaments of the temporomandibular joint. Part I: Ligamentum sphenomandibulare. Int J. Morphol., 32(3):981-6, 2014.

Ioannides, C. A. \& Hoogland, G. A. The disco-malleolar ligament: a possible cause of subjective hearing loss in patients with temporomandibular joint dysfunction. J. Maxillofac. Surg., 11(5):227-31, 1983.

Jufas, N.; Marchioni, D.; Tarabichi, M. \& Patel, N. Endoscopic anatomy of the protympanum. Otolaryngol. Clin. North Am., 49(5):1107-19, 2016.

Kim, H. J.; Jung, H. S.; Kwak, H. H.; Shim, K. S.; Hu, K. S.; Park, H. D.; Park, H. W. \& Chung, I. H. The discomallear ligament and the anterior ligament of malleus: an anatomic study in human adults and fetuses. Surg. Radiol. Anat., 26(1):39-45, 2004.

Komori, E.; Sugisaki, M.; Tanabe, H. \& Katoh, S. Discomalleolar ligament in the adult human. Cranio, 4(4):299-305, 1986.

Latarjet, M. \& Ruiz Liard, A. Anatomía Humana. Tomo 2. $2^{\mathrm{a}}$ ed. Buenos Aires, Médica Panamericana, 2005.

Loughner, B. A.; Gremillion, H. A.; Mahan, P. E. \& Watson, R. E. The medial capsule of the human temporomandibular joint. J. Oral Maxillofac. Surg., 55(4):363-9, 1997.

Loughner, B. A.; Larkin, L. H. \& Mahan, P. E. Discomalleolar and anterior malleolar ligaments: possible causes of middle ear damage during temporomandibular joint surgery. Oral Surg. Oral Med.Oral Pathol., 68(1):14-22, 1989.

Mérida-Velasco, J. R.; de la Cuadra-Blanco, C.; Pozo Kreilinger, J. J. \& Mérida-Velasco, J. A. Histological study of the extratympanic portion of the discomallear ligament in adult humans: a functional hypothesis. J. Anat., 220(1):86-91, 2012.

Mérida-Velasco, J. R.; Rodríguez, J. F.; de la Cuadra, C.; Peces, M. D.; Mérida, J. A. \& Sánchez, I. The posterior segment of the temporomandibular joint capsule and its anatomic relationship. J. Oral Maxillofac. Surg., 65(1):30-3, 2007.

Okeson, J. Tratamiento de Oclusión y Afecciones Temporomandibulares. $5^{\text {a }}$ ed. Madrid, Mosby, 2003.

Parker, W. S. \& Chole, R. A. Tinnitus, vertigo, and temporomandibular disorders. Am. J. Orthod. Dentofacial Orthop., 107(2):153-8, 1995.

Pinto, O. F. A new structure related to the temporomandibular joint and middle ear. J. Prosthet. Dent., 12(1):95-103, 1962.

Ramírez Aristeguieta, L. M.; Ballesteros Acuña, L. E. \& Sandoval Ortiz, G.P. A direct anatomical study of the morphology and functionality of disco-malleolar and anterior malleolar ligaments.Int.J.Morphol., 27(2) :367-79, 2009.

Ramírez, L. M.; Ballesteros, L. E. \& Sandoval, G. P. Síntomas óticos referidos en desórdenes temporomandibulares. Relación con músculos masticatorios. Rev. Med. Chile, 135(12):1582-90, 2007.
Rees, L. A. The structure and function of the mandibular joint. Br. Dent.J., 96:125-33, 1954.

Ricaurte, A. C. R. Informe de investigación: disección del ligamento discomaleolar en especímenes adultos, un niño y un feto. Univ. Odontol. 10(19):15-20, 1991

Rodríguez Vázquez, J. F.; Mérida Velasco, J. R. \& Jiménez Collado, J. Relationships between the temporomandibular joint and the middle ear in human fetuses. J. Dent. Res., 72(1):62-6, 1993.

Rodríguez-Vázquez, J. F.; Mérida-Velasco, J. R.; Mérida- Velasco, J. A. \& Jiménez-Collado, J. Anatomical considerations on the discomalleolar ligament. J. Anat., 192 (Pt. 4):617-21, 1998.

Rodríguez-Vázquez, J. F.; Murakami, G.; Verdugo-López, S.; Abe, S. \& Fujimiya, M. Closure of the middle ear with special reference to the development of the tegmen tympani of the temporal bone. J. Anat., 218(6):690-8, 2011

Rowicki, T. \& Zakrzewska, J.A study of the discomalleolar ligament in the adult human. Folia Morphol. (Warsz), 65(2):121-5, 2006.

Sencimen, M.; Yalçin, B.; Dog an, N.; Varol, A.; Okçu, K. M.; Ozan, H. \& Aydintug, Y.S. Anatomical and functional aspects of ligaments between the malleus and the temporomandibular joint. Int. J. Oral Maxillofac. Surg., 37(10):943-7., 2008

Toledo Filho, J. L.; Zorzetto, N. L. \& Navarro, J. A. Structures and relationships of the anterior malleus ligament. Anat. Anz., 158(1):1322, 1985.

Toller, M. O. \& Juniper, R. P. Audiological evaluation of the aural symptoms in temporomandibular joint dysfunction. J. Craniomaxillofac. Surg., 21(1):2-8, 1993.

Dirección para correspondencia

Prof. Dr. Ramón Fuentes Fernández

Centro de Investigación en Ciencias Odontológicas

Facultad de Odontología, Universidad de La Frontera

Av. Francisco Salazar 01145

Temuco

CHILE

E-mail: ramon.fuentes@ufrontera.cl

Recibido : 01-03-2018

Aceptado: 24-07-2018 